\title{
Bilateral ballism in a patient with overlapping Fisher's and Guillain-Barré syndromes
}

\author{
M Odaka, N Yuki, K Hirata
}

\begin{abstract}
A 29 year old woman developed diplopia and ataxic gait. Neurological examination showed total ophthalmoplegia, cerebellar ataxia, and areflexia. Moreover, there was muscle weakness in all four limbs. An overlap of Fisher's and Guillain-Barré syndromes was dignosed. On day 5 she suddenly developed involuntary flinging movements that affected the face and four limbs. Surface EMG showed 1.5-2 $\mathrm{Hz}$ rhythmic grouping discharges. The involuntary movements were considered ballism. This is the first report of a patient with Guillain-Barré syndrome and a related disorder who showed ballism. (F Neurol Neurosurg Psychiatry 1999;67:206-208)
\end{abstract}

Keywords: Bilateral ballism; Fisher's syndrome; Guillain-Barré syndrome; Bickerstaff's brainstem encephalitis

Fisher's syndrome, a monophasic illness of acute onset, is characterised by the triad of external ophthalmoplegia, cerebellar ataxia, and the absence of tendon reflexes. ${ }^{1}$ It is considered a variant form of Guillain-Barré syndrome because one third of patients with Fisher's syndrome eventually experience profound weakness. Myokimia ${ }^{2}$ and opsoclonus ${ }^{3}$ have been described in several cases of Guillain-Barré syndrome, but there have been no reports of ballism in either syndrome. We report a patient with overlapping Fisher's syndrome and Guillain-Barré syndrome who showed bilateral ballism.

\section{Case report}

A 29 year old woman with no history of antecedent infections developed diplopia and unsteady gait. On day 2, she had speech difficulties and could not stand up and was admitted to our hospital the same day. She was drowsy but roused easily on stimulation. Neuro-ophthalmological examination showed bilateral blepharoptosis, total opthalmoplegia, and incomplete convergence. The eyeballs were fixed in the central position, and there was no eye movement in any direction. Both pupils were dilated, with no reaction to light. Oculocephalic reflexes and Bell's phenomenon were absent. Neurological examination showed bilateral facial weakness and dysarthria, but corneal and gag reflexes were intact. She had no difficulty in swallowing, but elevation of the soft palate was limited. Proximal dominant weakness in the four limbs was 2 on the Medical Research Council scale. All the tendon reflexes were diminished or absent, and plantar responses were flexor. Because of severe trunkal ataxia, she could not stand. Muscle tone was decreased in all the limbs. Neither superficial nor deep sensation was affected. Autonomic nervous function was intact. There were no meningeal signs. The white blood count was $6600 / \mu 1$. A test for $\mathrm{C}$ reactive protein was negative. Blood glucose was $83 \mathrm{mg} / \mathrm{dl}$. Examination of the CSF on day 9 disclosed a raised protein concentration $(110 \mathrm{mg} / \mathrm{dl})$ with normal cellularity. On day 3 , her serum IgG anti-GQ1b antibody titre was high (64 000). Computed tomography and MRI with gadolinium enhancement showed no abnormality in the brainstem. This patient had the Fisher's syndrome triad. Moreover, the progression of muscle weakness in her limbs fulfilled the criteria for Guillain-Barré syndrome. These findings led to the diagnosis of overlapping Fisher's syndrome and Guillain-Barré syndrome. She underwent seven sessions of plasma exchange from day 3 to 17 . On day 4 , she developed dysphagia in the morning, and deglutition was impossible. Weakness of the limbs progressively worsened, and she became bedridden.

On day 5, she developed rapid involuntary flinging movements of both arms, combined with restlessness of the legs and grimacing facial expressions. These movements involved the proximal muscles of the shoulders and arms, and included abduction, adduction, internal and external rotation of the arms, and extension of the forearms (figure). Muscle tone in these areas was slightly reduced during rest. Movements were pronounced with intention, but disappeared with sleep. Her serum electrolytes, blood glucose concentration, and blood gas analysis results were within normal limits. Thyroid function studies and collagen vascular disease screens were negative. On day 6 , the movements became continuous, and on day 7 , they showed a slow, gradual decrease. On day 8 , the movements had almost completely disappeared. Repeated brain MRI on day 10 detected no abnormal findings in the subthalamic nucleus. 


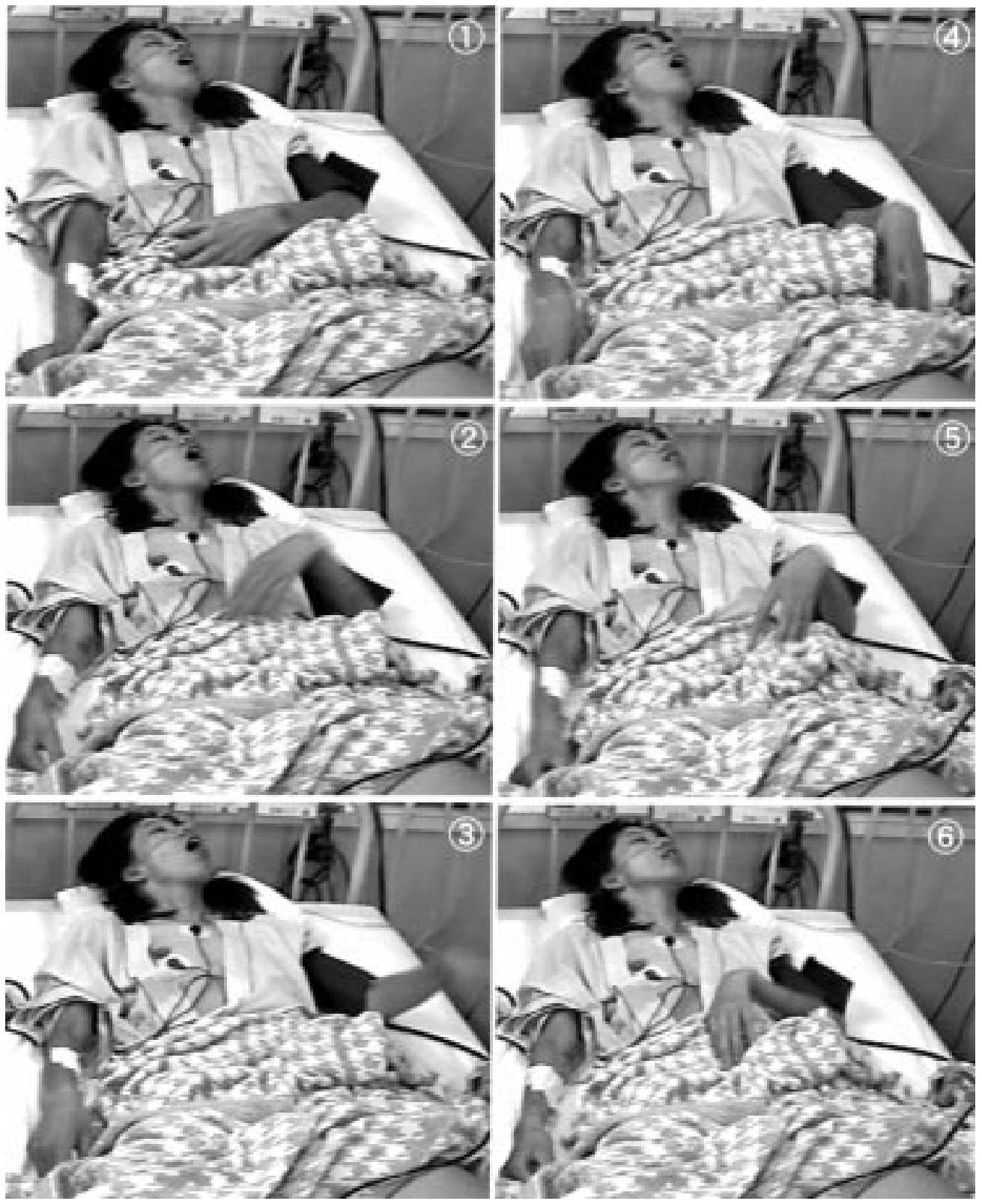

Photographs of the patient with bilateral ballism showing involuntary limbs and facial movement.

She began to recover consciousness on day 10 and was fully conscious on day 11 . On day 13 , she was able to stand with support, but walking was impossible. On day 24 , she could walk around the room with a wide based gait by holding onto the furniture. On day 19 , she was unable to swallow or to speak well. On day 17, there was slight movement of the eyeballs in downward gaze. Upward and horizontal movements began to return together, and the pupils were smaller and reacted briskly to the light. On day 40, horizontal nystagmus was present in the right and left gazes. On day 45 , as all motor weakness had disappeared and eye movements were limited only in abducting gaze, she was discharged from the hospital. Two months after onset, her range of eye movements was complete, and nystagmus had disappeared.

Surface EMG on day 7 showed $1.5-2 \mathrm{~Hz}$ rhythmic grouping discharges in the bilateral triceps and quadriceps femoris. Electroencephalography showed a bilateral diffuse spindle wave at 14 to $15 \mathrm{~Hz}$ while awake. On day 21 , blink reflex studies showed that on ipsilateral and contralateral stimulation $R_{2}$ latencies were bilaterally prolonged. On day 8 , auditory brainstem response showed prolonged interpeak latencies of the left III-IV waves, and absence of the left I-V waves. Visual evoked potentials and short latency somatosensory evoked potentials were normal. A nerve conduction study done on day 14 showed that the motor and sensory nerve conduction velocities were not reduced, only the compound muscle action potential in the right peroneal nerve had a low amplitude. An electronystagmogram on day 49 showed spontaneous nystagmus on lateral gaze, and an eye tracking test showed an ataxic pattern with impaired smooth pursuit eye movement. On day 23, an electroencephalogram was normal. 
On day 40, her auditory brainstem response and blink reflex had returned to normal.

\section{Discussion}

The movements made by this patient were quick flinging involuntary ones proximal in the four limbs and were synchronous with facial movements. Surface EMG recordings showed rhythmic grouping discharges. We consider that these movements were ballism, which consists of proximal, repetitive, stereotyped flinging movements of an arm or leg on one side of the body, usually caused by infarction of the subthalamic nucleus. ${ }^{4}$ There have been less than 20 reports of bilateral ballism. In 1986, Hoogstraten et al reviewed 13 cases. Seven of the patients had bilateral lesions in the basal ganglia with cerebrovascular disease. Since that review, other reports on bilateral ballism have defined its causes as oral contraceptives, ${ }^{6}$ multiple sclerosis, ${ }^{7}$ ventriculoperitoneal shunting, ${ }^{8}$ intravascular dissemination of gastric carcinoma, ${ }^{9}$ and non-ketotic hyperglycaemia. ${ }^{10}$ Ballism in Guillain-Barré syndrome has not previously been reported. Ours is the first report of a patient with Guillain-Barré syndrome and a related disorder who showed ballism.

We deduced the lesion from the neurological findings. The patient's drowsiness but easy rousing on stimulation suggests that the brainstem reticular formation was impaired. Fixation of the eyeballs in the central position, with no oculocephalic reflexes suggests the presence of nuclear or infranuclear lesions in the ocular motor nerves, and impaired smooth pursuit is indicative of cerebellar dysfunction. The clinical picture was characterised by a spindle wave on EEG. The spindle wave has been associated with lesions of the midbrain and subthalamic nucleus. ${ }^{11}$ The blink reflex suggests the localisation of the lesion, probably in the caudal part of the long loop of the blink reflex. The auditory brainstem response reflects the functional integrity of the brainstem auditory pathways. The absence of wave III may be due to abnormalities at any point from the superior olive to the inferior colliculus. Our patient's neurological signs and electrophysiological findings therefore are consistent with a lesion in the caudal brainstem, and cerebellum involvement.

Bickerstaff's brainstem encephalitis is characterised by acute ophthalmoplegia and ataxia with CNS signs and symptoms. Some patients with this disease show drowsiness, brisk reflexes, extensor plantar responses, hemisensory disturbance, or CSF pleocytosis. ${ }^{12}$ The predominant clinical features in our patient, including consciousness disturbance and ballism, are consistent with the diagnosis of overlapping Bickerstaff's brainstem encephalitis; however, as a patient with similar clinical features in association with case 2 was described by Fisher, ${ }^{1}$ we considered the diagnosis for our patient was Fisher's syndrome. IgG anti-GQ1b antibody is a possible immunological marker of Bickerstaff's brainstem encephalitis, as well as of Fisher's syndrome and Guillain-Barré syndrome with ophthalmoplegia. We have proposed elsewhere that Fisher's syndrome and Bickerstaff's brainstem encephalitis, variants of Guillain-Barré syndrome, represent a specific autoimmune disease that has a wide range of symptoms, including ophthalmoplegia and ataxia, because Bickerstaff's brainstem encephalitis and Fisher's syndrome are similar clinically and both have the IgG anti-GQ1b antibody. ${ }^{13} \mathrm{We}$ conclude that physicians should be aware of patients with Guillain-Barré syndrome with related disorders and ballism, and that these disorders could be useful in the choice of treatment with plasmapheresis.

1 Fisher M. An unusual variant of acute idiopathic polyneuritis: syndrome of ophthalmoplegia, ataxia and areflexia. $N$ tis: syndrome of ophthalmople
Engl $\mathcal{F}$ Med 1956;255:57-65.

2 Mateer JE, Gutmann L, McComas CF. Myokymia in Guillain-Barré syndrome. Neurology 1983;33:374-6.

3 Nicolai A, Lazzarino LG. Opsoclonus in a patient with Guillain-Barré syndrome. Acta Neurol Scand 1992;85:3634.

4 Buruma OJS, Lakke JPWF. Ballism. In: Vinken PJ, Bruyn GW, Klawans HL, eds. Handbook of clinical neurology. Vo 49. Amsterdam: North-Holland, 1986:369-80.

5 Hoogstraten MC, Lakke JPWF, Zwarts MJ. Bilateral ballism: a rare syndrome. $\mathcal{F}$ Neurol 1986;233:25-9.

6 Driesen JJ, Wolters EC. Bilateral ballism induced by oral contraceptives: a case report. $\mathcal{F}$ Neurol 1986;233:379.

7 Masucci EF, Saini N, Kurtzke JF. Bilateral ballism in multiMasucci EF, Saini N, Kurtzke JF. Bilatera
ple sclerosis. Neurology 1989;39:1641-3.

8 Walker FO, Hunt VP. Ballism. an association with ventriculo-peritoneal shunting. Neurology 1990;40:1004

9 Laing RW, Howell SJL. Acute bilateral ballism in a patient with intravascular dissemination of gastric carcinoma. Neuropathol Appl Neurobiol 1992;18:201-5.

10 Lai PH, Tien RD, Chang MH, et al. Chorea-allismus with nonketotic hyperglycemia in primary diabetes mellitus. Am f Neuroradiol 1996;17:1057-64.

11 Steudel WI, Krüger J, Grau H. Zur alpha-und spindelaktivität bei komatösen patienten nach einer schädel-HirnVerletzung unter besonderer berücksichtigung der computertomographie. Z EEG-EMG 1979;10:143-7.

12 Bickerstaff ER. Brain-stem encephalitis: further observaBickerstaff ER. Brain-stem encephalitis: further observa-
tions on a grave syndrome with benign prognosis. $B M \mathcal{F}$ $1957 ; \mathrm{i}: 1384-7$

13 Yuki N, Sato S, Tsuji S, et al. An immunologic abnormality common to Bickerstaff's brain stem encephalitis and Fisher's syndrome. $\mathcal{F}$ Neurol Sci 1993;118:83-7. 